\title{
CrystEngComm
}

Check for updates

Cite this: CrystEngComm, 2020, 22, 2845

Received 21st February 2020

Accepted 25th March 2020

DOI: $10.1039 /$ d0ce00263a

rsc.li/crystengcomm

\section{Synthesis of all-silica hollow zeolites by selective demetallation $\uparrow$}

\author{
Teng Li, (iD ${ }^{a}$ Frank Krumeich (iD ${ }^{a}$ and Jeroen A. van Bokhoven (iD *ab
}

We report a method to efficiently produce all-silica hollow zeolites by leaching their parent crystals in sodium aluminate and hydrochloric acid solutions successively. The extra-framework aluminum species resulted from the dissolution of sodium aluminate can protect the crystal rim from damaging, and are subsequently eliminated by acid wash.
Materials with hollow structure find interest in catalysis due to their key advantages compared to their counterparts with non-hollow morphologies. ${ }^{1,2}$ They effectively encapsulate and isolate metal nanoparticles from each other and thus prevent sintering under harsh catalytic conditions. Besides, they are efficient in case mass transfer plays a role since the shell can be made very thin. If the shell part possesses a regular pore structure, such as in the case of hollow zeolites, features of size-selectivity and poison-resistance for catalysts can be achieved. ${ }^{3,4}$

With respect to the synthesis of hollow zeolite crystals, different synthetic routes have been explored, such as by using sacrificial templates ${ }^{5,6}$ or exploiting zeolite crystallization patterns. ${ }^{7,8}$ A recent trend to synthesize hollow zeolites is synthesizing zeolite crystals first and then introducing hollow structures by selectively removing the core $_{\text {part. }}{ }^{9-13}$ Through this facile route, hollow zeolite crystals with a single crystalline shell can be produced. Exploiting the aluminum zoning in ZSM-5 zeolites, hollow crystals can be prepared by leaching the corresponding parent crystals in a mildly alkaline solution. ${ }^{14,15}$ This is attributed to the negative charge of the $\mathrm{AlO}_{4}{ }^{-}$tetrahedra in the shell framework that protect the mircroporous structure. Silicate zeolites attract lots of attention due to their high thermal stability and hydrophobicity compared to their aluminosilicate counterparts. The absence of aluminum-related acid sites also suppress the undesired reactions such as the coking reaction. However, with regards to synthesizing all-silica hollow crystals, the most reported efficient method is to use a tetrapropylammonium hydroxide (TPAOH) solution to leach crystals at the crystallization temperature of zeolites. ${ }^{16-20}$

\footnotetext{
${ }^{a}$ Institute for Chemical and Bioengineering, ETH Zurich, 8093 Zurich, Switzerland. E-mail: jeroen.vanbokhoven@chem.ethz.ch; Tel: +41446325542

${ }^{b}$ Paul Scherrer Institute, 5232 Villigen, Switzerland

$\dagger$ Electronic supplementary information (ESI) available: Experimental details, characterization data and complementary images included. See DOI: 10.1039/ doce00263a
}

TPAOH acts as both the alkaline solution to etch the core part, as well as the organic structure directing agent (OSDA) to direct any crystallization of the leached species on the shell. As the same with other OSDAs, TPAOH is costly, possibly leading to an increase of the cost in the preparation of all-silica hollow zeolites. Therefore, other leaching reagents and procedures need to be explored.

Herein, we report a method to prepare all-silica hollow zeolite crystals by successive desilication and dealumination with low-cost reagents. Compared to the TPAOH route to obtain hollow crystals, the present protocol involving desilication and dealumination is less energy-intensive due to a low temperature operation. The key is using sodium aluminate with suitable concentrations to leach the zeolite crystals to first obtain the hollow structure. Then, an acid wash allows to eliminate the extra-framework aluminum species.

Fig. 1a and b show electron micrographs of the $\mathrm{S} 1$ (the defect-rich silicalite-1) crystals which were synthesized in basic media. Crystals have a hexagonal shape of size between 200 and $300 \mathrm{~nm}$. Recently, based on this material we

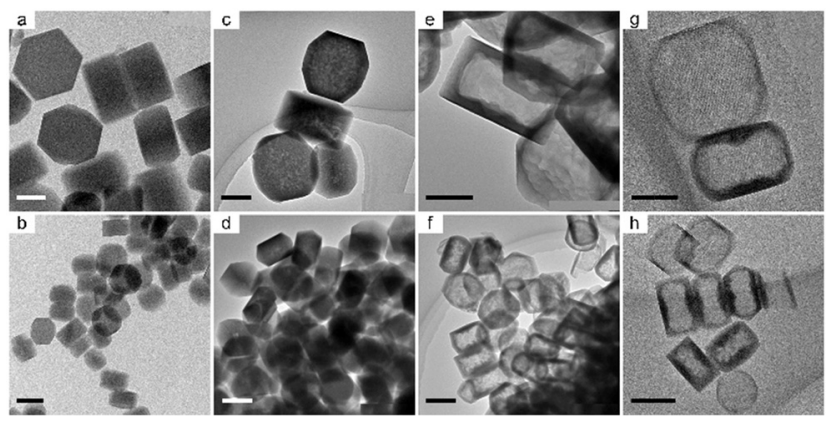

Fig. 1 TEM images of ( $a$ and $b$ ) the parent crystals $S 1$, (c and d) the base leached crystals S1-0.01 M, (e and f) the base leached hollow crystals S1-0.1 $\mathrm{M}$ and ( $\mathrm{g}$ and $\mathrm{h}$ ) the acid leached hollow crystals S1-0.1 $\mathrm{M}-\mathrm{HCl}$. S1 refers to silicalite-1 sample with abundant inner defects. Scale bars are $100 \mathrm{~nm}$ for ( $a, c$, e and g) and $200 \mathrm{~nm}$ for (b, d, $f$ and h). 


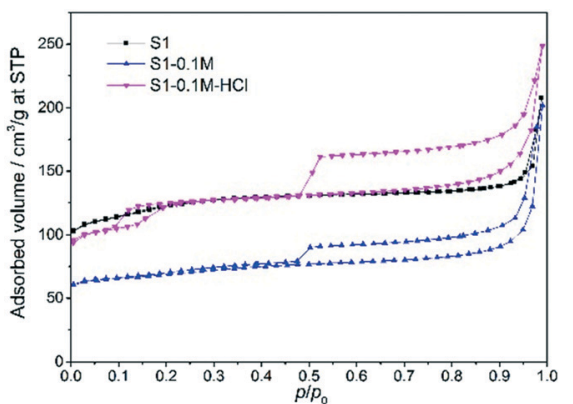

Fig. 2 Nitrogen physisorption isotherms for the parent crystals (S1), and the leached crystals (S1-0.1 M, S1-0.1 M-HCl).

reported a procedure to visualize the uneven distribution of defects by using a heavy atom labeling method in conjunction with electron microscopy. ${ }^{21}$ The presence of abundant inner defects allows the preferential desilication of the defective core part, which is essential to making the hollow structures. The S1 crystals were firstly leached in a dilute sodium aluminate solution $(0.01 \mathrm{M})$ at $60{ }^{\circ} \mathrm{C}$. A period of 4 hour leaching introduced mesopores only to the core part of the crystals (Fig. 1c and d). Close examination of the crystals (S1-0.01 M) indicates that their shell part was relatively intact, suggesting that an increase of base concentration may be feasible to obtain hollow structures. Fig. 1e and $\mathrm{f}$ show the images of the leached crystals by 0.1 M sodium aluminate solution (S1-0.1 M), where hollow structures were observed. Different from the previously reported hollow crystals, the S1-0.1 M crystals possessed a rough surface, implying the presence of non-zeolite relics. A further increase of the base concentration $(0.5 \mathrm{M})$ lead to both the severe damage of crystals and the generation of amorphous material (Fig. S1†).

To remove the non-zeolite relics, the S1-0.1 M crystals were subsequently leached in $0.3 \mathrm{M}$ hydrochloric acid solution. Crystals $(\mathrm{S} 1-0.1 \mathrm{M}-\mathrm{HCl})$ with more pronounced hollow structures were obtained, with an average shell thickness of $25 \mathrm{~nm}$ (Fig. $1 \mathrm{~g}$ and $\mathrm{h}$ ). The preservation of crystallinity is confirmed by powder X-ray diffraction (Fig. S2†).

Fig. 2 shows the nitrogen physisorption isotherms of the samples and Table 1 lists the derived parameters. S1 had a relatively large surface area $\left(S_{\mathrm{BET}}=424 \mathrm{~m}^{2} \mathrm{~g}^{-1}\right)$, which decreased significantly after leaching the samples in 0.1 sodium aluminate solution $\left(S_{\mathrm{BET}}=238 \mathrm{~m}^{2} \mathrm{~g}^{-1}\right)$. In the meantime, the micropore volume decreased by $36 \%$, which

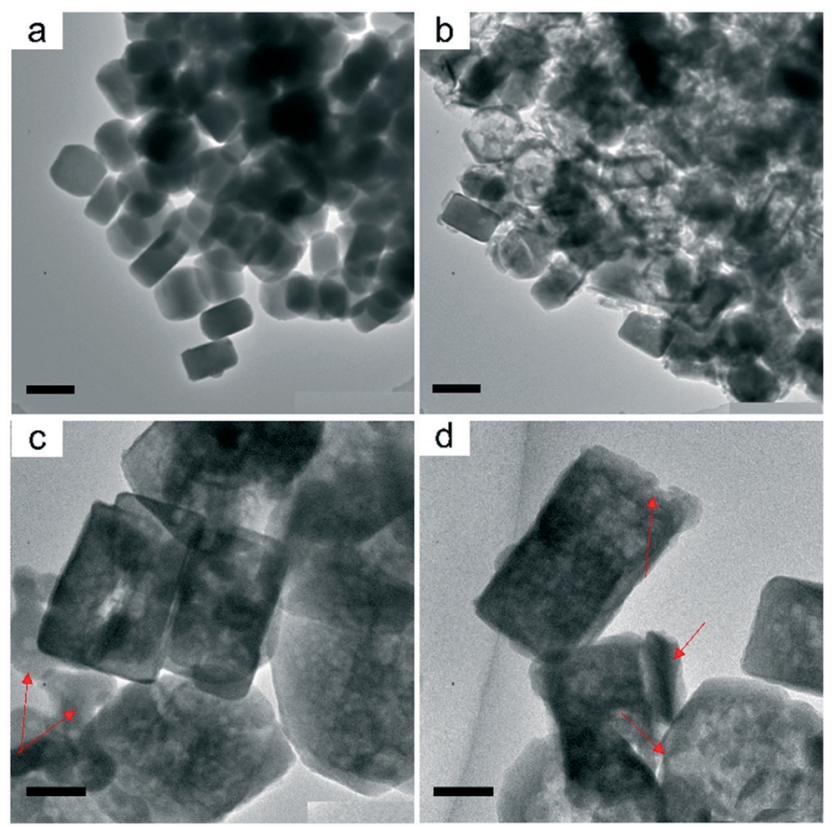

Fig. 3 TEM images of the leached crystals by (a) $0.1 \mathrm{M}$ sodium carbonate, $\mathrm{pH}=11.3$, (b) $0.1 \mathrm{M}$ sodium hydroxide, $\mathrm{pH}=12.8$, and (c and d) $0.025 \mathrm{M}$ sodium hydroxide, $\mathrm{pH}=12.3$ which is close to the $\mathrm{pH}$ value of $0.1 \mathrm{M}$ sodium aluminate 12.3. The red arrows in (c) and (d) indicate the amorphous relics and the damaged rim part, respectively. Scale bars are $200 \mathrm{~nm}$ for ( $a$ and b) and $50 \mathrm{~nm}$ for (c and d).

can be explained by the presence of non-zeolite species after leaching. Elemental analysis indicated that such species had a high aluminum content since the $\mathrm{Si} / \mathrm{Al}$ ratio of the samples decreased to 17 (Table 1). The ${ }^{27} \mathrm{Al}$ MAS NMR spectrum of S1$0.1 \mathrm{M}$ shows a single signal at about $60 \mathrm{ppm}$ that can be assigned to tetrahedrally coordinated aluminum, and no octahedral aluminum was observed (Fig. S3†). The peak center of resonance was different from that of aluminum in ZSM-5 zeolite $(53 \mathrm{ppm})^{15}$ and sodium aluminate $(80 \mathrm{ppm})$, indicative of the presence of non-zeolite aluminum species. Interestingly, the aluminum-containing species are homogeneously distributed in the shell part (Fig. S4a and $\mathrm{b} \dagger)$. An acid wash efficiently eliminated the aluminumcontaining species, as confirmed by elemental analysis (Table $1, \mathrm{Si} / \mathrm{Al}$ ratio is infinity), the noisy signals in both ${ }^{27} \mathrm{Al}$ MAS NMR spectrum (Fig. S3†) and energy-dispersive X-ray spectroscopy (EDX) mapping (Fig. S4c and di). As a result, both the surface area and micropore volume of the samples were restored, and a more pronounced hysteresis loop of S1$0.1 \mathrm{M}-\mathrm{HCl}$ compared to that of S1-0.1 M suggests the hollow

Table $1 \mathrm{Si} / \mathrm{Al}$ ratio and derived parameters obtained from $\mathrm{N}_{2}$ physisorption isotherms

\begin{tabular}{|c|c|c|c|c|c|}
\hline Sample & $\mathrm{Si} / \mathrm{Al}^{a}$ & $S_{\mathrm{BET}}^{b}\left[\mathrm{~m}^{2} \mathrm{~g}^{-1}\right]$ & $S_{\text {ext }}^{c}\left[\mathrm{~m}^{2} \mathrm{~g}^{-1}\right]$ & $V_{\text {tot }}{ }^{d}\left[\mathrm{~cm}^{3} \mathrm{~g}^{-1}\right]$ & $V_{\text {micr }}{ }^{c}\left[\mathrm{~cm}^{3} \mathrm{~g}^{-1}\right]$ \\
\hline $\mathrm{S} 1-0.1 \mathrm{M}$ & 17 & 238 & 77 & 0.19 & 0.07 \\
\hline
\end{tabular}

${ }^{a}$ Determined by AAS. ${ }^{b}$ Derived from the BET model. ${ }^{c}$ Derived from the $t$-plot method. ${ }^{d}$ Derived from single point at $p / p_{0}=0.96 . S_{\mathrm{BET}}, S_{\mathrm{ext}}, V_{\mathrm{tot}}$ and $V_{\text {micr }}$ represent the surface area, the external surface area, the total pore volume and the micropore volume, respectively. 

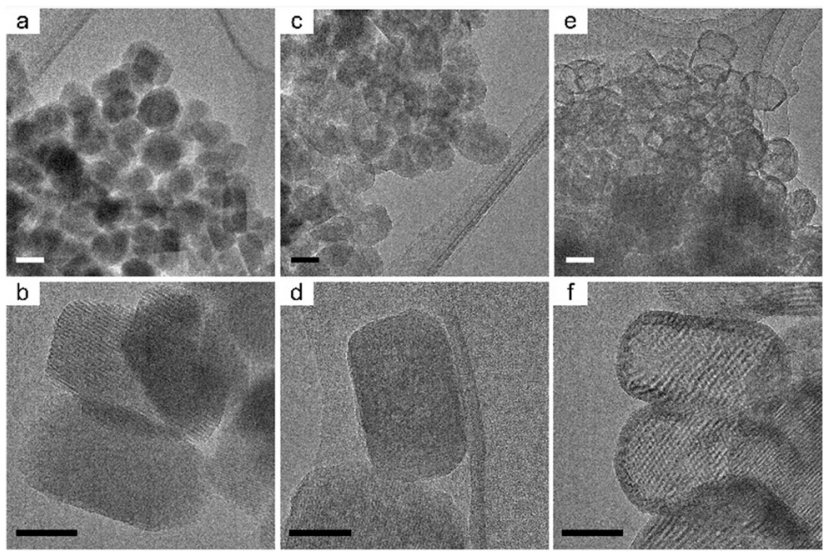

Fig. 4 TEM images of ( $a$ and $b$ ) the parent crystals S2, (c and d) the base leached crystals S2-0.1 M, (e and f) the base leached crystals S20.2 M. S2 refers to silicalite- 1 sample with less inner defects. Scale bars are $100 \mathrm{~nm}$ for ( $\mathrm{a}, \mathrm{c}$ and $\mathrm{e}$ ) and $50 \mathrm{~nm}$ for (b, d and f).

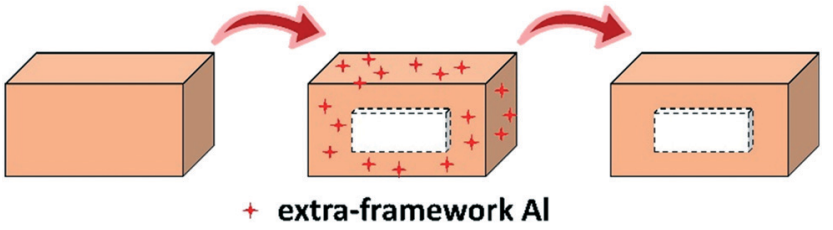

Scheme 1 Illustration for the formation of all-silica hollow zeolite crystals by successive desilication and dealumination.

structure and relatively smooth outer surface. The sub-step in the range of $p / p_{0}=0.1-0.2$ is attributed to a phase transition of the adsorbed $\mathrm{N}_{2}$ from a disordered "fluid" phase to a more ordered "crystalline" like phase, which has been observed in MFI zeolites with high Si/Al ratio and less defects. ${ }^{22-24}$ Considering the parent silicalite- 1 crystals possess abundant defects in the core and less defects in the rim, we can deduce that the resulted hollow counterparts would exhibit the feature of sub-step.

To prove the role of defects in directing the mesopore formation, a time-series leaching experiment was performed. In various time-points, mixtures of pristine, partially leached, and fully leached particles can be observed (Fig. S5†). In general, the leaching started from the core, and more and more mesopores were generated until they coalesced into a big cavity. The preferential elimination of the core should be related to the abundant silanol defect inside of zeolites, as confirmed by Fourier-transform infrared spectroscopy (Fig. S6 $\dagger$ ). The presence of silanol nests $\left(3500 \mathrm{~cm}^{-1}\right)$ and the obvious external silanol peak $\left(3726 \mathrm{~cm}^{-1}\right)$ implied the existence of abundant defects.

Aluminum-zoning is well-known for zeolite ZSM-5, allowing the preparation of their hollow crystals by different leaching reagents. This is mainly attributed to the protective function of framework aluminum in the shell part. In addition, the extra-framework aluminum species, as created by the steaming ${ }^{25}$ or the extra addition of aluminum nitrate to an alkaline solution, ${ }^{26,27}$ also inhibit silicon extraction. Verboekend $e t$ al. found that the metal complexes $\left(\mathrm{Al}(\mathrm{OH})_{4}{ }^{-}\right.$, $\left.\mathrm{Ga}(\mathrm{OH})_{4}{ }^{-}\right)$avoided the excessive dissolution of silicalite- 1 crystals, although their target is to obtain crystals with randomly distributed mesopores. ${ }^{26}$ To explore the function of extra-framework aluminum species in the present procedure, we compared the leaching results based on different reagents. Fig. 3a shows the TEM image of the crystals leached in $0.1 \mathrm{M}$ sodium carbonate. No mesopores were observed, which could be due to the low $\mathrm{pH}$ value (11.3). Leaching crystals in $0.1 \mathrm{M}$ sodium hydroxide lead to the generation of hollow structures, but the shell part was severely damaged. Besides, some crystal were not leached well, with fewer mesopores (Fig. 3b). To study the effect of $\mathrm{pH}$ on desilication, a sodium hydroxide solution with lower concentration $(0.025$ M) was prepared, which had a pH value (12.3), similar to that of $0.1 \mathrm{M}$ sodium aluminate. Compared to S1-0.1 M (Fig. 1e and f), the leaching of crystals in $0.025 \mathrm{M}$ sodium hydroxide was inefficient, as the hollowfication degree of crystals was much lower, suggesting insufficient $\mathrm{OH}^{-}$in desilication (Fig. 3c and d). Moreover, both the presence of a large portion of amorphous species and the damaged shell were observed. Considering the reversible attribute of the sodium aluminate dissolution, a slow release of $\mathrm{OH}^{-}$was expected, which could explain its lower initial $\mathrm{pH}$ value but providing sufficient $\mathrm{OH}^{-}$during the whole desilication process. In total, the aluminum-deposited species resulted from the sodium aluminate can suppress the attack of $\mathrm{OH}^{-}$ on the zeolite shell.

Next, we extend the procedure to a silicalite-1 sample with fewer inner defects, namely S2 (defect-poor silicalite-1). ${ }^{14}$ The introduction of S2 to this study is used to prove that the protective effect of aluminum-containing species plays a more important role than the content of defects in forming hollow crystals. Crystals had a size between 100 and $150 \mathrm{~nm}$ (Fig. 4a and b). After leaching in $0.1 \mathrm{M}$ sodium aluminate, only mesopores were observed (Fig. 4c and d). A further increase of sodium aluminate concentration to $0.2 \mathrm{M}$ sodium aluminate results in the generation of the hollow structure, which is confirmed by the obvious contrast between the core and the shell of crystals. The feasibility of using sodium aluminate solutions with suitable concentrations in producing all-silica hollow zeolites are substantiated in both the defect-rich and the defect-poor silicalite- 1 crystals.

In summary, as illustrated in Scheme 1, by using sodium aluminate and hydrochloric acid solutions successively to leach all-silica zeolites, we could synthesize all-silica hollow crystals in an economic route. The dissolution of sodium aluminate provides not only hydroxide ions to attack the defective core part of crystals, but also extra-framework aluminum species to protect the shell part of crystals. An eventual acid wash allows to efficiently eliminate the aluminum-containing species from the zeolite part, and subsequently well crystalline all-silica hollow crystals are obtained.

The authors thank Scientific Center for Optical and Electron Microscopy of the Swiss Federal Institute of Technology (ETH) for technical support. Teng Li thanks the China Scholarship Council (CSC) for financial support (201506450010). 


\section{Conflicts of interest}

The authors declare no conflict of interest.

\section{Notes and references}

1 G. Prieto, H. Tüysüz, N. Duyckaerts, J. Knossalla, G.-H. Wang and F. Schüth, Chem. Rev., 2016, 116, 14056-14119.

2 W. Zhu, Z. Chen, Y. Pan, R. Dai, Y. Wu, Z. Zhuang, D. Wang, Q. Peng, C. Chen and Y. Li, Adv. Mater., 2018, 1800426.

3 C. Pagis, A. R. Morgado Prates, D. Farrusseng, N. Bats and A. Tuel, Chem. Mater., 2016, 28, 5205-5223.

4 A. Tuel and D. Farrusseng, Small Methods, 2018, 2, 1800197.

5 V. Valtchev, Chem. Mater., 2002, 14, 4371-4377.

6 N. Chu, J. Wang, Y. Zhang, J. Yang, J. Lu and D. Yin, Chem. Mater., 2010, 22, 2757-2763.

7 X. Chen, M. Qiao, S. Xie, K. Fan, W. Zhou and H. He, J. Am. Chem. Soc., 2007, 129, 13305-13312.

8 T. Li, F. Krumeich and J. A. Van Bokhoven, Cryst. Growth Des., 2019, 19, 2548-2551.

9 C. Mei, Z. Liu, P. Wen, Z. Xie, W. Hua and Z. Gao, J. Mater. Chem., 2008, 18, 3496-3500.

10 T. Li, J. Ihli, J. T. Wennmacher, F. Krumeich and J. A. van Bokhoven, Chem. - Eur. J., 2019, 25, 7689-7694.

11 J. C. Groen, T. Bach, U. Ziese, A. M. Paulaime-van Donk, K. P. de Jong, J. A. Moulijn and J. Pérez-Ramírez, J. Am. Chem. Soc., 2005, 127, 10792-10793.

12 C. Pagis, A. R. M. Prates, N. Bats, A. Tuel and D. Farrusseng, CrystEngComm, 2018, 20, 1564-1572.
13 A. R. M. Prates, T. Chetot, L. Burel, C. Pagis, R. MartinezFranco, M. Dodin, D. Farrusseng and A. Tuel, J. Solid State Chem., 2020, 281, 121033.

14 T. Li, H. Wu, J. Ihli, Z. Ma, F. Krumeich, P. H. Bomans, N. A. Sommerdijk, H. Friedrich, J. P. Patterson and J. A. Van Bokhoven, J. Mater. Chem. A, 2019, 7, 1442-1446.

15 T. Li, Z. Ma, F. Krumeich, A. J. Knorpp, A. B. Pinar and J. A. Van Bokhoven, ChemNanoMat, 2018, 4, 992-999.

16 Y. Wang, M. Lin and A. Tuel, Microporous Mesoporous Mater., 2007, 102, 80-85.

17 S. Li, L. Burel, C. Aquino, A. Tuel, F. Morfin, J. Rousset and D. Farrusseng, Chem. Commun., 2013, 49, 8507-8509.

18 C. Dai, A. Zhang, L. Li, K. Hou, F. Ding, J. Li, D. Mu, C. Song, M. Liu and X. Guo, Chem. Mater., 2013, 25, 4197-4205.

19 S. Li, T. Boucheron, A. Tuel, D. Farrusseng and F. Meunier, Chem. Commun., 2014, 49, 8507-8509.

20 C. Dai, X. Li, A. Zhang, C. Liu, C. Song and X. Guo, RSC Adv., 2015, 5, 40297-40302.

21 T. Li, F. Krumeich, J. Ihli, Z. Ma, T. Ishikawa, A. B. Pinar and J. A. van Bokhoven, Chem. Commun., 2019, 55, 482-485.

22 J. C. Groen and J. Pérez-Ramírez, Appl. Catal., A, 2004, 268, 121-125.

23 J. C. Groen, L. A. A. Peffer and J. Pérez-Ramírez, Microporous Mesoporous Mater., 2003, 60, 1-17.

24 Y. Wang and A. Tuel, Microporous Mesoporous Mater., 2008, 113, 286-295.

25 J. C. Groen, L. A. Peffer, J. A. Moulijn and J. Pérez-Ramírez, Chem. - Eur. J., 2005, 11, 4983-4994.

26 D. Verboekend and J. Pérez-Ramírez, Chem. - Eur. J., 2011, 17, 1137-1147.

27 J. Li, M. Liu, X. Guo, S. Zeng, S. Xu, Y. Wei, Z. Liu and C. Song, Ind. Eng. Chem. Res., 2018, 57, 15375-15384. 